\title{
Active Acquisition of Information for Diagnosis and Supervisory Control of Discrete Event Systems
}

\author{
David Thorsley • Demosthenis Teneketzis
}

Published online: 16 October 2007

CC Springer Science + Business Media, LLC 2007

\section{Erratum to: Discrete Event Dyn Syst DOI 10.1007/s10626-007-0027-y}

In (Thorsley and Teneketzis 2007), Definition 1 is incomplete. The revised definition below states the additional necessary condition missing from Thorsley and Teneketzis (2007).

Definition 1 An observation policy $g:=\left(g_{0}, \ldots, g_{T-1}\right)$ is a sequence of functions $g_{t}$ : $L_{T} \rightarrow 2^{\Sigma_{c o}}$ such that for all $t, t=0, \ldots, T-1, g_{t}$ is measurable with respect to the $\sigma$-field $\mathcal{G}_{t}^{g}$, defined below (in Definition 3).

Note that for all $s^{\prime} \in L_{t}$ and $s, \hat{s} \in \chi_{t}\left(s^{\prime}\right), g_{t}(s)=g_{t}(\hat{s})$. The statement "The functions $\chi_{t}$ are used in the following definition," found above Definition 1 in the text, should be ignored when reading the paper.

Definition 3 is unchanged, but we restate it here for convenience.

The online version of the original article can be found at http://dx.doi.org/10.1007/s10626-007-0027-y

D. Thorsley $(\varangle)$

Department of Electrical Engineering, University of Washington, Seattle, WA 98195, USA

e-mail: thorsley@ee.washington.edu

D. Teneketzis

Department of EECS, University of Michigan, Ann Arbor, MI 48109, USA

e-mail: teneketzis@eecs.umich.edu 
Definition 3 The filtration $\left\{\mathcal{G}_{t}^{g}, t=0 \ldots T\right\}$ corresponding to $g$ is

$$
\sigma\left(\pi_{t}: \pi_{t} \in R_{t}^{g}\right), t=0 \ldots T .
$$

Note that $\mathcal{G}_{t}^{g}, t=0 \ldots T-1$, depends on $g_{0}, g_{1}, \ldots, g_{t-1}$.

\section{References}

Thorsley D, Teneketzis D (2007) Active acquisition of information for diagnosis and supervisory control of discrete event systems. Discret Event Dyn Syst: Theory and Applications 17(4): $531-583$ 